\title{
Investigation of neutron emission by $n-n$ correlation function in fission process using an array detector
}

\author{
I. Companis ${ }^{\text {a }}$, A. Isbasescu, M. Mirea, and H. Petrascu
}

Horia Hulubei National Institute for Physics and Nuclear Engineering, P. O. Box MG-6, 077125 Bucharest-Magurele, Romania

\begin{abstract}
An experimental set-up designed to investigate the origin of the prompt scission neutrons in fission processes by using an array neutron detector is modeled. Quantities of interest as the correlation function strength in the momentum space within a model of independent one-particle pointlike sources, with a Gaussian distribution for the neutron generation points are obtained in a preliminary investigation. Two working hypothesis are used: $i$ ) the scission neutrons are emitted from the neck between fragments, in the final stage of the fission process, and $i$ ) the post-scission neutrons are emitted from the accelerated fission fragments. For the scission neutrons emitted from the neck, a n-n correlation strength significantly larger than that obtained for neutrons emitted from the fission fragments is obtained. The results indicate that it is possible to discriminate scission and post-scission neutrons within an time-of-flight experimental set-up consisting from an array neutron detector combined with a VME acquisition system, using ( $\mathrm{p}, \mathrm{xnf})$ reactions on actinide targets at the Bucharest Tandem.
\end{abstract}

\section{Introduction}

Investigation of prompt neutrons emission is a challenging issue pertaining to fundamental aspects of the nuclear fission process. It is known that fission is accompanied by prompt neutrons, but their origin is still uncertain. Most of these neutrons, called post-scission neutrons, are emitted from the fully accelerated Fission Fragments (FF). Some of them are emitted from the excited fissioning nucleus, before fission occurs, called pre-scission neutrons or in the moment of rupture, called scission neutrons (10-15\%) [1]. The experimental results concerning the emission of scission or pre-fission neutrons are rather contradictory due to some arbitrary assumptions made in various analyses. Recently, an approach dealing with fission induced by polarized neutrons has been proposed to solve this situation [1, 2] exploiting the dependence between the neutron spatial orientation and the FF flight direction. This approach is extremely difficult mostly because of the reduced events statistics.

An alternative method could be the correlation analysis of the prompt neutrons in the fission process [3]. As it is known the correlation function is very sensitive to the space-time dimension from which the neutrons are emitted. Until now the method of pair momentum correlations of identical particles has been successfully used by us in the investigation of the neutron pair pre-emitted from Boromean halo nuclei [4-6]. This method allows to determine the space-time characteristics of the particle pairs source and to distinguish between the post-scission and scission neutrons due to the strength of the n-n correlation function. Other important characteristics in the investigation of prompt

\footnotetext{
a e-mail: companis@nipne.ro
}

fission neutrons emission are the neutron multiplicity and the neutron spectrum. These observables lead to a better understanding of the nucleon-nuclei interaction mechanisms and the fission process properties.

Our purpose is to investigate the prompt fission neutrons emission in ${ }^{233,235,238} \mathrm{U}(\mathrm{p}, \mathrm{xnf})$ reactions for energy beams $E_{p}$ of $6-16 \mathrm{MeV}$ provided by the Bucharest Tandem accelerator. It is advantageous to work with proton beam because consistent information concerning the investigated reactions may be more easily obtained. An experimental set-up consisting on time of flight array neutron detector combined with a VME acquisition system will be used the first time for this purpose. This array neutron detector consisting of 81 scintillator cells, initially build for neutron pairs pre-emitted from halo nuclei investigations, has been adapted to work conditions of Bucharest Tandem.

\section{Method}

The correlation technique is based on the principle that the wave function of the relative motion of identical particles emitted from a source of a certain space-time configuration is modified through the Final State Interaction (FSI) and by the symmetries of quantum statistics (QSS)[7]. Starting from 1954, when the stellar interferometry was discovered through the effect Hanbury-Brown-Twiss (HBT), the method was adopted for interferometry in high energy particles physics. Later, the interferometry method was developed in nuclear physics, focusing on particles pairs correlations. Theoretical improvements in the analysis of the correlation functions were realized by the Dubna group [8, 9] and developed in $[10,11]$ by taking into account the FSI.

This is an Open Access article distributed under the terms of the Creative Commons Attribution-Noncommercial License, which permits unrestricted use, distribution, and reproduction in any noncommercial medium, provided the original work is properly cited. 


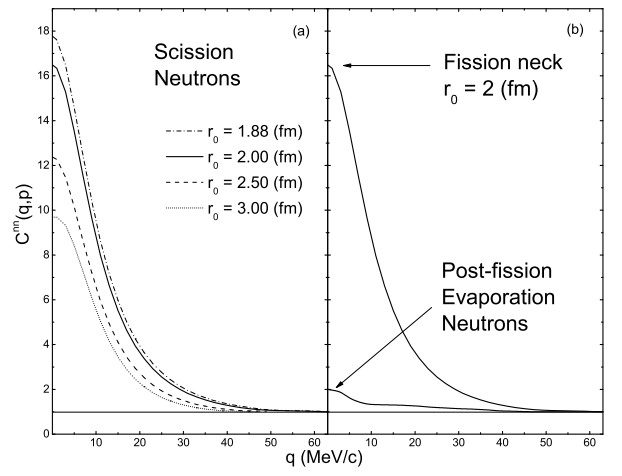

Fig. 1. $C_{n n}(q)$ for prompt fission neutrons as a function of relative momentum $q(\mathrm{MeV} / \mathrm{c})$. (a) $C_{n n}$ for scission neutrons for different values of $r_{0}$. (b) Comparison between $C_{n n}$ for scission neutrons and $C_{n n}$ for neutrons emitted from fully accelerated FF.

In the following, the analytical approach $[10,11]$ that describe the neutron narrow pair correlations at small relative momenta $q$, within a model of single-particle pointlike sources, taking into account the Fermi statistics and the s-wave strong final state interaction will be used. The neutron generation points are assumed independent and their distribution is considered Gaussian. The normalized correlation function of two neutrons with momenta $p_{1}=$ $\left\{E_{1}, \mathbf{p}_{1}\right\}$ and $p_{2}=\left\{E_{2}, \mathbf{p}_{2}\right\}$ is defined as the ratio of two particle spectrum (the yield of coincidence events) to the product of respective one-particle spectra (the yield of uncorrelated events), $C_{n n}(q, p)=k N_{c}(q, p) / N_{n c}(q, p)$ where $q=\left(p_{1}-p_{2}\right) / 2$ and $p=\left(p_{1}+p_{2}\right) / 2$. As mentioned, the method has been successfully used by us to investigate the neutron pairs pre-emitted from ${ }^{11} \mathrm{Li}$ halo nuclei, using an array neutron detector $[4,5]$. Thereby, it was possible to distinguish between the neutron pairs pre-emitted from ${ }^{11} \mathrm{Li}$ halo nuclei and the neutrons emitted from the fusion process. In this context, the $C_{n n}$ theoretical value for the neutrons pairs pre-emitted from ${ }^{11} \mathrm{Li}$ halo nuclei had the value 10, while the experimental one was 7-8. This last value is affected by residual correlations. For neutrons emitted in the fusion process this value reached 2 [6]. Motivated by these results, we consider that the method of $n-n$ correlations is also adequate in the fission studies, in order to separate the scission neutrons from the post-scission neutrons. As predicted by hydrodynamic models, the fission neck between FF in the final state configurations behaves as a filament of neutron matter. A few number of prompt neutrons strongly correlated could be emitted in the moment when the two FF are separated, while for postscission neutrons the n-n correlations are almost absent.

The calculations realized in the present work take into account the experimental data for ${ }^{233,235,238} \mathrm{U}$ (p,xnf) [12$14]$, in correlation with the most probable fragmentation [15]. This fragmentation is obtained from the weighted mass numbers using relation (2) of Ref. [13]. The most probable charge $Z_{p H}$ and $Z_{p L}$ for heavy and light fragments, respectively, is considered by mean of the unchanged charge division distribution assumption given by formulas (4) and (5) of Ref. [16]. In order to calculate the correlation function for post-scission neutrons, the root mean square radius for each light and heavy FF is also obtained by exploiting the most probable fragmentation method. The average prompt neutron multiplicity and the neutron spectrum of the fission process are obtained in the frame of the Los Alamos (LA) model [15, 17, 18].

\section{Results and discussion}

\subsection{Estimation of the $C_{n n}$ strength for scission prompt neutrons}

Using the analytical formula (24) of Ref. [10] for nonrelativistic neutrons, we calculated $C_{n n}(q, p)$. It is assumed that the scission neutrons are emitted from a neck formed between the fragments, in the final state of the fission process [3]. In Fig. 1, the dependence of $C_{n n}(q, p)$ versus the relative moment $q$ of the neutron pairs is illustrated, $r_{0}$ being the variance of the Gaussian distribution [10,11]. A scattering length of the two neutrons in the singlet state $f_{0}=17 \mathrm{fm}$ and an effective range $d_{0}=2.7 \mathrm{fm}$ are considered. The distance between the 2 neutrons is given by the root mean square radius $r_{s q}=\sqrt{3} r_{0}$. We performed this calculation for different $r_{0}$ values ranging from (2-3) fm. These values are justified by calculations of the tip distance between fragment at scission in the frame of a microscopicmacroscopic model applied to ${ }^{236} \mathrm{~Np}$, the Compound $\mathrm{Nu}$ cleus $(\mathrm{CN})$ obtained from ${ }^{235} \mathrm{U}(\mathrm{p}, \mathrm{f})$. The tip distance between the fission fragments at scission for the fissioning nucleus ${ }^{236} \mathrm{~Np}$ has been evaluated [19]. We started from a nuclear shape parametrization characterized by 5 degrees of freedom, one being related to the curvature of the neckingin $C$, and another one being the elongation given by the distance between the centers of the fragments denoted $R$. To obtain the scission configuration, the fission trajectory in the five-dimensional configuration space is determined by minimizing the action integral. Plots of the minimal deformation energy surface as function of the necking coordinate $C$ and the elongation $R$ are displayed in Figs. 2 (a) and (b). The resulting ${ }^{236} \mathrm{~Np}$ fission barrier is plotted on Fig. 2 (c) as function of the distance between the centers of the nascent fragments. From the exit of the barrier, the potential energy is positive and the parent nucleus is able to collapses into two separated fragments. So, it is possible to appreciate the tip distance between the two fragments by making difference between the elongation in the exit point of the barrier and the dimensions of the two formed nuclei. The tip distance obtained in this way is $3 \mathrm{fm}$. In Fig. 1(a) it is observed that the n-n correlation strength has the maximum intensity for $r_{0}=1.88 \mathrm{fm}$ while for $r_{0}=2 \mathrm{fm}$ is 17.5. A significant difference between maximum value for $n-n$ correlation function for prompt scission neutrons and for post-fission neutrons is obtained. This difference can be exploited to separate the scission and post-scission neutrons.

\section{$3.2 C_{n n}$ calculation for post-scission neutrons.}

The correlation function $C_{n n}$ for neutrons emitted from fully accelerated FF where the the Fermi statistics contribution 

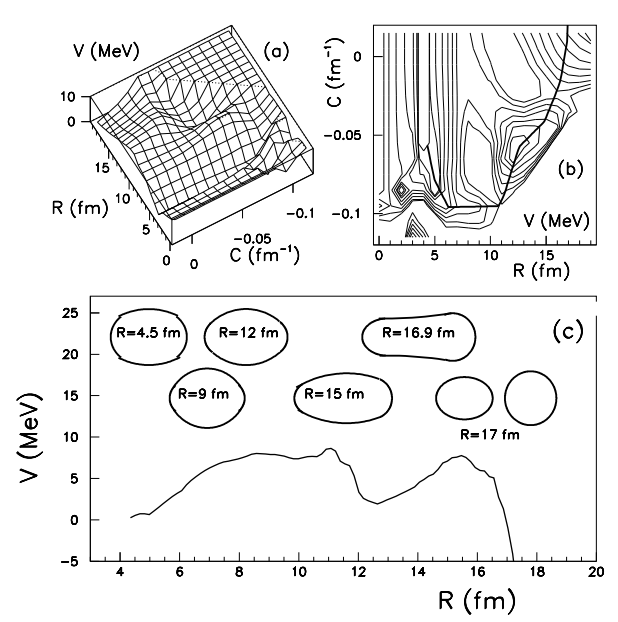

Fig. 2. (a) Minimal values of the deformation energy in $\mathrm{MeV}$ as function of the necking coordinate $C$ and the elongation $R$ for ${ }^{236} \mathrm{~Np}$. (b) Contours of the deformation energy in step of $1 \mathrm{MeV}$. The least action trajectory is superimposed. (c) Potential barrier. Some shapes obtained during the fission process together with the values of the elongation $R$ are inserted.

is dominant, was determined using Eqs. (17) and (25) of Ref. [10]. The root mean square radius was calculated for each light and heavy FF taken into consideration. For the fissioning nucleus ${ }^{236} \mathrm{~Np}$ the $\mathrm{r}^{r m s}$ for light fragment (Zr$101)$ is $2.91 \mathrm{fm}$ and $\mathrm{r}^{r m s}$ for heavy fragment (I-135) is 3.21 $\mathrm{fm}$. The FF masses were chosen according to the most probable fragmentation method using the experimental data [12-14]. The correlation strength for post-scission neutrons is $\leq 2$. In Fig. 1(b) the correlation strength $C_{n n}$ in the case of neutron emission from the neck, at the scission point, exceeds by far the value corresponding to post-scission evaporation neutrons. Due to this difference in $C_{n n}$ the two mechanisms involved could be separated, scission neutrons from the neck and post-scission neutrons emission from fully accelerated FF. We highlight that the $n-n$ correlation function strength for neutron emitted from Borromean halo nuclei is around 10, while for neutrons resulting from the fission neck, in the final state of the fission process, the correlation strength is around 20. This last value is significantly higher than that obtained in the case of neutron emitted from fully accelerated FF which is around 2. This difference is mainly caused by the strong correlations that characterize the few scission neutrons. The correlation is practically absent for the large number of post-fission evaporation netrons. Another argument in favor of the n-n correlation function measurement is the following: the prompt neutrons emitted from the neck between the FF require a duration of emission of the order of $10^{-22} \mathrm{~s}$, while the emitted neutrons from the excited fission fragments require times of order $10^{-19} \mathrm{~s}$ [20]. This time difference can be measured within the $C_{n n}$ method and causes modifications in the correlation intensity. Thus, the two processes can be very well distinguished when they are unfolded as in the case of halo nuclei.

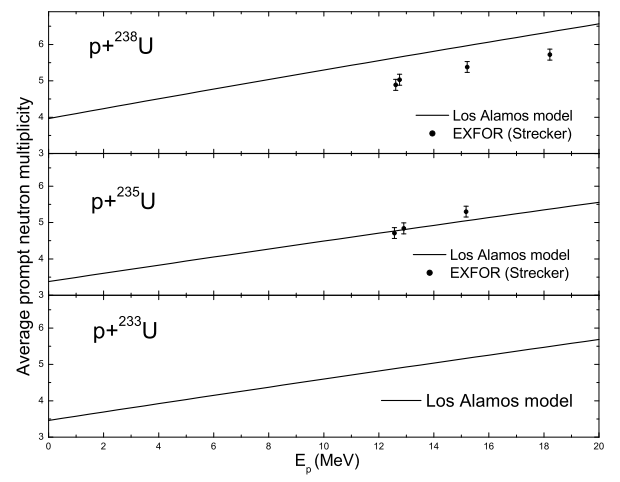

Fig. 3. Average prompt neutron multiplicity for $\mathrm{p}+{ }^{233,235,238} \mathrm{U}$ versus the incident proton energy and the experimental data.

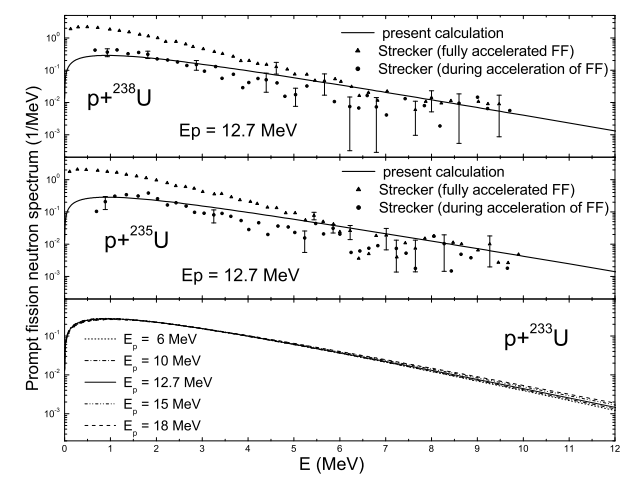

Fig. 4. The prompt neutron fission spectra of ${ }^{234,236,239} \mathrm{~Np}$ isotopes at different proton incident energies $E_{p}$ compared with the experimental data.

\subsection{Average total neutron emission}

In this subsection the proton induced fission on the three uranium isotopes ${ }^{233,235,238} \mathrm{U}$ is addressed. The prompt neutron spectrum and the average prompt neutron multiplicity are calculated at an incident proton energies ranging between $0.1-20 \mathrm{MeV}$, exploiting the LA model. Values of the model parameters required for the calculation of the average prompt neutron multiplicities and the fission neutron spectra are presented in Table I. The average energy release $\left\langle E_{r}>\right.$ is determined by using the mass excess for the entire FF range. The values of the proton separation energy $B_{p}$ and also the average neutron separation energy $\left.<S_{n}\right\rangle$ were calculated according to Ref. [21]. For the average total kinetic energy we used formula (2) from Ref. [22]. The average prompt gamma energy $\left\langle E_{\gamma}\right\rangle$ was determined semi-empirically as $E_{\gamma}=0.7<S_{n}>$ [23]. The energy dependent $\mathrm{CN}$ cross section for representative average central light and heavy fragments corresponding to each CN, was obtained using Becchetti-Greenless optical potential [24]. Calculated average neutron multiplicities as function of the proton incident energy for ${ }^{234,236,239} \mathrm{~Np}$ are displayed in Fig. 3 together with experimental data [12, 23]. In Fig. 3, a good agreement between experimental data for the $\mathrm{p}+{ }^{235} \mathrm{U}$ reaction and our simulations is evidenced in the evaluated energy range. Similar behaviours of the average multiplicity energy dependence are also obtained for $\mathrm{p}+{ }^{238} \mathrm{U}$. For the other analyzed reactions, the 
EPJ Web of Conferences

Table 1. Average values of the parameters used for calculating the prompt fission neutron spectra and average prompt neutron multiplicities for the reactions studied in the present work.

\begin{tabular}{llllllll}
\hline $\begin{array}{l}\text { Fission } \\
\text { Reaction }\end{array}$ & $\begin{array}{l}\text { Average } \\
\text { Light Fragment }\end{array}$ & $\begin{array}{l}\text { Average } \\
\text { Heavy Fragment }\end{array}$ & $\begin{array}{l}B_{p} \\
(\mathrm{MeV})\end{array}$ & $\begin{array}{l}\left\langle E_{r}\right\rangle \\
(\mathrm{MeV})\end{array}$ & $\begin{array}{l}\langle T K E\rangle \\
(\mathrm{MeV})\end{array}$ & $\begin{array}{l}\left\langle S_{n}\right\rangle \\
(\mathrm{MeV})\end{array}$ & $\begin{array}{l}\left\langle E_{\gamma}\right\rangle \\
(\mathrm{MeV})\end{array}$ \\
\hline${ }^{233} \mathrm{U}(\mathrm{p}, \mathrm{xnf})$ & ${ }^{99} \mathrm{Zr}$ & ${ }^{135} \mathrm{I}$ & 4.25 & 201.51 & 174.31 & 6.21 & 4.34 \\
${ }^{235} \mathrm{U}(\mathrm{p}, \mathrm{xnf})$ & ${ }^{101} \mathrm{Zr}$ & ${ }^{135} \mathrm{I}$ & 4.83 & 200.61 & 173.82 & 6.41 & 4.48 \\
${ }^{238} \mathrm{U}(\mathrm{p}, \mathrm{xnf})$ & ${ }^{103} \mathrm{Zr}$ & ${ }^{136} \mathrm{I}$ & 5.28 & 197.18 & 173.11 & 4.97 & 3.48 \\
\hline
\end{tabular}

experimental data are scarce or totally missing (like the case of $\left.\mathrm{p}+{ }^{233} \mathrm{U}\right)$, therefore it is not possible to appreciate the agreement of the theoretical predictions. Some discrepancies can be caused by the choice of the most probable fragmentation that leads to the average values of the parameters of the model. In Fig. 4, the prompt neutron fission spectra of ${ }^{234,236,239} \mathrm{~Np}$ isotopes at different incident energies $E_{p}=6,10,12.7,15,18 \mathrm{MeV}$ are represented. The evaluated data of $\mathrm{p}+{ }^{235,238} \mathrm{U}$ are in agreement with the sole available experimental data at $E_{p}=12.7 \mathrm{MeV}$. The experimental data were obtained by digitizing the experimental points of Ref. [14] with cartographic accuracy. For $p+{ }^{233} U$ the experimental data are completely missing. A systematic analysis of average prompt neutron multiplicity and specrum will be presented in a forthcoming paper.

Recently a VME data acquisition system used with the array neutron detector mentioned previously has been installed at the Bucharest Tandem accelerator and was tested using the proton induced fission reaction on ${ }^{235} \mathrm{U}$ target. The array neutron detector, with 81 scintillator cells was built in IFIN-HH and tested at the RIKEN-RIPS separator within radioactive beams. It has been successfully used in the investigation of the pair neutron pre-emission from ${ }^{11} \mathrm{Li}$ halo nuclei and has been adapted for Tandem conditions [25]. The work is in progress.

\subsection{Conclusions}

The strength of correlation n-n functions $C_{n n}$ for scission and post-scission neutrons are calculated. The strength of the $C_{n n}$ at small relative momenta has very large values in the case of scission neutrons. The model used takes into account the final state interaction dominant in the case of scission neutrons and the Fermi statistic contribution important in the case of post-scission neutrons. A significant difference between the $n-n$ correlation function intensity obtained for prompt scission neutrons and for post-fission neutrons was evidenced. Due to this difference, the scission and post-scission neutrons could be precisely separated.

The LA model was used to simulate average fission neutron multiplicities and spectra in proton induced fission reactions.

\section{Acknowledgments}

This work is dedicated to the memory of Prof. Dr. Marius Petrascu (1929-2007), personality devoted to nuclear physics research.
We are grateful to A. Pop, A. Tudora and A. Constantinescu for fruitful discussions. One of the authors (I.C.) would like to acknowledge the CNR*09 Organizing Committee for the grant obtained to attend the Conference. Work supported by CNCSIS IDEI 535 Contract.

\section{References}

1. G.A. Petrov et al., Phys. At. Nucl., 71, (2008) 1137

2. G.V. Danilyan et al.,Phys. At. Nucl., 69, (2006) 1158

3. M. Petrascu, Book of Abs., Int. Conf. 'Nucleus 2007', June 25-29, Voronezh, Russia (2007) 59

4. M. Petrascu et al., Phys. Rev. C 69, (2004) 011602

5. M. Petrascu et al., Phys. At. Nucl. 69, (2006) 1261

6. M. Petrascu et al., Nucl. Phys. A 790, (2007) 235c

7. D.H. Boal et al., Rev. Mod. Phys. 62, (1990) 553

8. G.I. Kopylov and M.I. Podgoretsky, Sov. J. Nucl. Phys. 15,(1972) 219

9. G.I. Kopylov, Phys. Lett. B 50, (1974) 472

10. R. Lednicky, V.L. Lyuboshitz, Sov. J. Nucl. Phys. 35, (1982) 770

11. V.L. Lyuboshitz and V.V. Lyuboshitz, Phys. At. Nucl.

71, (2008) 454

12. http://www-nds.iaea.org/EXFOR

13. T. Ohtsuki et al., Phys. Rev. C 44, (1991) 1405

14. T. Ohtsuki et al., Phys. Rev. C 40, (1989) 2144

15. D. Madland and R. Nix, Nucl. Sci. Eng. 81, (1982) 213

16. H. Naik et al., Nucl. Phys. A 781, (2007) 1

17. D. G. Madland, Nucl. Phys. A 772, (2006) 113

18. G. Vladuca, A. Tudora, Comput. Phys. Commun. 125, (2000) 221

19. I. Companis, M. Mirea and A. Isbasescu, in preparation

20. Yu.D. Katarzhnov et al., Phys. At. Nucl. 64, (2001) 177

21. G. Audi, A.H. Wapstra and C. Thibault, Nucl. Phys. A 729, (2003) 337

22. Y. Nagame et al., Rom. Rep. Phys. 59, (2007) 547

23. M. Strecker et al., Phys. Rev. C 41, (1990) 2172

24. O. Bersillon, SCAT2 optical model code, OECDNEA-DB-CPS 0829/03, (1991)

25. H. Petrascu et al., Rom. J. Phys., in press. 